\title{
BMJ Open Hypertensive pregnancy complications in women with epilepsy and antiepileptic drugs: a population-based cohort study of first pregnancies in Norway
}

Kim Christian Danielsson, ${ }^{1,2}$ Ingrid Borthen, ${ }^{2}$ Nils-Halvdan Morken, ${ }^{1,3}$ Nils Erik Gilhus ${ }^{2,4}$

To cite: Danielsson KC, Borthen I, Morken $\mathrm{N}-\mathrm{H}$, et al. Hypertensive pregnancy complications in women with epilepsy and antiepileptic drugs: a population-based cohort study of first pregnancies in Norway. BMJ Open 2018;8:e020998. doi:10.1136/ bmjopen-2017-020998

- Prepublication history for this paper is available online. To view these files, please visit the journal online (http://dx.doi. org/10.1136/bmjopen-2017020998).

Received 6 December 2017 Revised 27 March 2018 Accepted 28 March 2018

\section{Check for updates}

${ }^{1}$ Department of Obstetrics and Gynecology, Haukeland University Hospital, Bergen, Norway

${ }^{2}$ Department of Clinical Medicine, University of Bergen, Bergen, Norway

${ }^{3}$ Department of Clinical Science, University of Bergen, Bergen,

Norway

${ }^{4}$ Department of Neurology, Haukeland University Hospital, Bergen, Norway

\section{Correspondence to} Dr Kim Christian Danielsson; kim.christian.danielsson@helsebergen.no

\section{ABSTRACT}

Objectives To estimate the risk of hypertensive pregnancy complications in women with epilepsy, with and without antiepileptic drugs, and assess the risk associated with the four most common antiepileptic drugs.

Design A population-based cohort study using linked data from the Medical Birth Registry of Norway and the Norwegian Prescription Database. Women with epilepsy with and without antiepileptic drugs were compared with women without epilepsy.

Setting Norway, 2004-2012.

Participants All first pregnancies of women with epilepsy and women without epilepsy were included.

Primary and secondary outcome measures Main outcome measures were hypertensive pregnancy complications: a compound variable of any hypertensive disorder, gestational hypertension, mild pre-eclampsia, severe pre-eclampsia, early onset pre-eclampsia, eclampsia and HELLP syndrome (haemolysis, elevated liver enzymes, low platelets).

Results In total, 1778 pregnancies in women with epilepsy and 221662 in women without epilepsy were analysed. 682 of the women with epilepsy used antiepileptic drugs, the most common in monotherapy being: lamotrigine $(n=280)$, carbamazepine $(n=94)$, levetiracetam $(n=71)$ and valproate $(n=51)$. There was an increased risk of any hypertensive disorder in women with epilepsy (adjusted OR (aOR) 1.2, 95\% Cl 1.0 to 1.5) and in the subcategory using valproat $(\mathrm{aOR} 2.9,95 \% \mathrm{Cl} 1.3$ to 6.4). The most frequent hypertensive complication was mild pre-eclampsia and the risk was increased in women with epilepsy (aOR 1.4, 95\% $\mathrm{Cl} 1.1$ to 1.8) and women with epilepsy with valproat (aOR $3.3,95 \% \mathrm{Cl} 1.2$ to 9.4 ).

Conclusions Women with epilepsy have an increased risk of mild pre-eclampsia, but not for the severe types of hypertensive pregnancy complications. Lamotrigine and levetiracetam do not predispose for mild pre-eclampsia, whereas valproate was associated with an increased risk of mild pre-eclampsia.

\section{INTRODUCTION}

Epilepsy is the most common neurological disorder requiring continuous drug
Strengths and limitations of this study

- Large population-based national cohort with data from compulsory and reliable national health registries.

- Only first pregnancies were included to avoid bias from recurrent events.

- We have studied type and severity of hypertensive complications in pregnancy and associations with use of specific antiepileptic drugs.

We had no data on type of epilepsy or seizure activity.

treatment during pregnancy, and 30\%-70\% of pregnant women with epilepsy (WWE) use antiepileptic drugs (AEDs). ${ }^{1-4}$ Studies have focused on consequences of epilepsy and maternal AED use during pregnancy for fetal malformations and child development. ${ }^{5-8}$ In contrast, potential effects of epilepsy and specific AEDs on the pregnancy have been less studied. Optimal therapy in epilepsy is a balance between seizure suppression and side effects. For women in fertile age, potential AED effects for the fetus as well as on the pregnancy should be of the highest interest.

Hypertensive pregnancy complications include multiple diagnoses with different risks and implications for fetal and maternal outcomes. Hypertensive pregnancy complications represent a major contributor to global maternal mortality. ${ }^{9}$ Most previous studies, including a recent meta-analysis, have found an increased risk of total hypertensive complications in WWE, but these studies have not differentiated between the specific hypertensive disorders and therefore the burden of disease in WWE is uncertain. ${ }^{2310-14}$ Studies on the effect of specific AEDs on pregnant WWE have been limited by small sample size, selection bias and lack of detailed information on 
hypertensive disorders. ${ }^{2}{ }^{10-13} 15$ Recently, a small cohort study found an increased risk of pre-eclampsia in WWE with the newer AED lamotrigine. ${ }^{3}$ Some studies have also suggested an increased pre-eclampsia risk with the older AEDs, carbamazepine and valproate. ${ }^{12} 13$ Many AED work by blocking ion channels, enhancing gamma-aminobutyric acid receptors or blocking glutamate receptors, and they stabilise and inactivate neurons. Valproate can induce endocrine changes and metabolic syndrome in WWE and possibly also hypertensive complications in pregnancy. ${ }^{16}$ No other AED mode of action is known to pose any increased risk for hypertension or pre-eclampsia. Preferred drugs and drugs to be avoided in pregnancy have not been identified, despite an increased use in young women of the newer AEDs lamotrigine and levetiracetam. ${ }^{1718}$

The aim of the present study was to estimate the risk of specified hypertensive pregnancy complications in WWE with and without use of AEDs, and to specifically define the risk for the four most common AEDs. This is necessary to guide optimal AED treatment in young women before and during pregnancy.

\section{MATERIALS AND METHODS}

We used data from the compulsory, population-based Medical Birth Registry of Norway (MBRN) collected during 2004-2012. The notification form of the MBRN was unchanged in that period. MBRN includes data on maternal and paternal social factors, maternal health prior to and during pregnancy including chronic diseases such as epilepsy, complications and interventions during pregnancy and birth, perinatal outcome and complications in the children. Data are prospectively registered throughout pregnancy, delivery and postpartum period. All data are forwarded to the MBRN by the attending midwife or obstetrician. All pregnancies from 12 weeks gestation are recorded. The registry is routinely linked to the National Population Database to ensure notification of all births and deaths in the country. All Norwegian citizens are given a unique Social Security Number that enables linkage of data from various national data sources. Only first time pregnancies were included in this study, all singleton and multiple pregnancies at $>22$ weeks of gestation.

The Norwegian Prescription Database (NorPD), commenced in 2004, provides detailed data on all medications dispensed from pharmacies by prescription. Mandatory registration in NorPD of every dispensed medical prescription is made by the pharmacist. Statistics Norway provided data on maternal level of education.

Main outcome variables were a compound variable of any hypertensive disorder in pregnancy, gestational hypertension, mild pre-eclampsia, severe pre-eclampsia, early onset pre-eclampsia, eclampsia and HELLP syndrome (haemolysis, elevated liver enzymes, low platelets). Exposure variables were a diagnosis of epilepsy and use of AEDs. All hypertensive disorders, except the compound variable, are reported in MBRN in specified checkboxes. Checkboxes are not mutually exclusive. Therefore, we used the most severe diagnosis to exclude double registration. Gestational hypertension was defined as persisting elevated blood pressure $\geq 140 \mathrm{~mm} \mathrm{Hg}$ systolic and/or $\geq 90 \mathrm{~mm} \mathrm{Hg}$ diastolic occurring after 20 weeks of gestation and without pre-existing hypertension. ${ }^{19}$ Mild pre-eclampsia was defined as persisting elevated blood pressure $\geq 140 \mathrm{~mm} \mathrm{Hg}$ systolic and/or $\geq 90 \mathrm{~mm} \mathrm{Hg}$ diastolic combined with proteinuria $\geq 0.3 \mathrm{~g}$ per 24 hours (equivalent to $\geq+1$ on urine test strips for protein excretion) occurring after 20 weeks of gestation. Severe pre-eclampsia was defined as blood pressure $\geq 160 / 110 \mathrm{~mm} \mathrm{Hg}$, clinical symptoms of pre-eclampsia, protein excretion $\geq 3 \mathrm{~g}$ per 24 hours or oliguria and also included all cases of early onset pre-eclampsia ( $<34$ weeks), eclampsia or HELLP syndrome. Eclampsia was defined as generalised seizures occurring simultaneously with pre-eclampsia or hypertension and without other cause. Early onset pre-eclampsia was defined as pre-eclampsia with onset before 34 weeks of gestation. The compound variable of any hypertensive disorder was defined as the presence of any of these hypertensive disorders. Superimposed pre-eclampsia in women with pregestational hypertension was not specified but was included in the respective subcategories of hypertensive complications by severity.

Maternal epilepsy was defined by either ticking the specific checkbox in MBRN or adding this diagnosis in a blank space available for written text. Neither of these provided information about the type of epilepsy or seizure activity. The diagnosis was based on previous medical history and medical charts. The epilepsy diagnosis in MBRN has previously been found to be valid in $92.3 \%$ of cases, having been confirmed in hospital medical records. $^{3}$

NorPD provided exact data on dispensed AEDs and high-dose folic acid supplementation $(4 \mathrm{mg} /$ day) including type of medication (according to Anatomical Therapeutic Chemical Classification system), dose and time of administration. We defined AED use in pregnancy as dispensed drugs from the pharmacy in the time period coinciding with gestation, dated by ultrasound assessment. In cases with missing ultrasound dates, last menstrual period was used. We assessed drug dispensation also 1 and 3 months preconception. An a priori decision was made to primarily analyse the four most commonly used AEDs in monotherapy in order to obtain large enough groups and exclude interference of polytherapy. Information about AED use in pregnancy is also recorded in MBRN on the notification form in an available blank space for written text. This information was used to validate the AED use obtained from MBRN compared with NorPD. Positive predictive values for AED data in MBRN were calculated by matching with confirmed AED dispensation in NorPD during pregnancy. The positive predictive values were 0.94 for lamotrigine, 0.82 for carbamazepine, 0.95 for levetiracetam and 0.84 for valproate, providing acceptable registration. When AED use in NorDP data 
was assessed 1 and 3 months before conception, the total AED population remained unchanged. Ninety-five per cent of WWE with AED in NorPD had more than one dispensation during pregnancy, illustrating continuous use throughout pregnancy as one dispensation usually corresponds to more than 3 months use.

Sociodemographic and background data included maternal age ( $\leq 19$ years, $20-24$ years, $25-29$ years, $30-34$ years, 35-39 years and $\geq 40$ years), maternal educational level ( $\leq 10$ years, $11-13$ years, $\geq 14$ years), other chronic disease (kidney disease, hypertension, diabetes mellitus), multifetal pregnancies, high pregestational body mass index (BMI) (BMI $\geq 30 \mathrm{~kg} / \mathrm{m}^{2}$ ), smoking during pregnancy and use of folic acid supplementation in standard dose $(0.4 \mathrm{mg} /$ day $)$. The BMI variable was only available from 2006 onwards. Data on ethnicity and marital status were not available for this study.

We further categorised WWE by AED treatment; all AEDs, no AEDs, AED polytherapy and the four most common AEDs. Outcome variables for specific AEDs were analysed only in WWE with monotherapy. WWE using other than the four most common AEDs were identified and excluded from the analyses where appropriate. WWE and all treatment subcategories were compared with women without epilepsy. WWE with AED treatment were also compared with WWE without AEDs.

For binary outcomes, proportions and crude ORs were calculated using contingency tables. $\mathrm{P}$ values were calculated using Fisher's exact test. Continuous variables (background variables) were analysed with independent sample t-tests. Statistical significance was defined as non-overlapping 95\% CIs and two-sided $\mathrm{p}$ values $<0.05$. All statistical analyses were performed with IBM SPSS V.22.0 or later.

We used logistic regression to adjust for possible confounding by maternal age, educational level, multifetal pregnancy, use of folic acid supplements and other relevant chronic diseases (pre-existing hypertension, pre-existing kidney disease and pre-existing diabetes mellitus). The covariates BMI (only registered since 2006) and smoking were restrained by missing cases and therefore studied with stratification-based sensitivity analyses applied on all results and not included in the final logistic regression models. The impact of other relevant chronic diseases was similarly analysed further with stratification-based sensitivity analyses.

The MBRN, NorPD and Statistics Norway approved the use of data. Use of Norwegian national register data does not require consent from the participants.

\section{Patient involvement}

The Norwegian Epilepsy Association provided input to the research questions in this study.

\section{RESULTS}

A total of 223440 first-time pregnancies were eligible after exclusion of multipara pregnancies (309 364), missing personal identification number (1514, $0.7 \%$ (foreigners or immigrants without residence permit)) and pregnancies <23 gestational weeks $(340,0.2 \%)$. There were 1778 WWE $(0.79 \%)$, of whom 682 (38.4\% of WWE) used AEDs either in monotherapy (554 women) or in polytherapy (128 women). The most common AEDs used as monotherapy were lamotrigine (280 women, $50.5 \%$ of all monotherapies), carbamazepine (94 women, $17.0 \%$ ), levetiracetam (71 women, $12.8 \%$ ) and valproate (51 women, 9.2\%). Other AEDs in monotherapy were used by 58 WWE. WWE were younger, had lower educational level, were more often obese, smoked more often and were more likely to have comorbidity compared with women without epilepsy. All maternal characteristics are shown in table 1.

The adjusted OR (aOR) of the compound variable any hypertensive disorder was increased in WWE (aOR 1.2, $95 \%$ CI 1.0 to 1.5) compared with women without epilepsy. However, the occurrence of any hypertensive disorder in neither the group WWE with AED nor the group WWE without AED differed significantly from women without epilepsy when examined separately.

The majority of hypertensive complications were mild pre-eclampsia (table 2).

The risk of mild pre-eclampsia was significantly increased in WWE (aOR 1.4, 95\% CI 1.1 to 1.8 ) and in WWE without AED exposure (aOR 1.4, 95\% CI 1.0 to 2.0) compared with women without epilepsy. For WWE with AED the risk of mild pre-eclampsia was not significantly increased although the prevalence was the same as for both WWE and WWE without AEDs. For WWE, there was a marginally significant risk of early onset pre-eclampsia (aOR 1.7, 95\% CI 1.0 to 2.8). This risk disappeared after stratification and when including smoking or other relevant chronic diseases in sensitivity analyses. Mild pre-eclampsia was therefore the only specified hypertensive disorder increased in WWE. There was no significant difference for any of the outcomes when WWE with AEDs were compared with WWE without AED. There was no significantly increased risk of gestational hypertension, severe pre-eclampsia, HELLP or eclampsia when WWE, WWE with and WWE without AED were compared with women without epilepsy.

WWE with valproate monotherapy had an increased risk of any hypertensive complication (aOR 2.9, 95\% CI 1.4 to 6.4 ), gestational hypertension (aOR $3.3,95 \%$ CI 1.0 to 10.8 ) and mild pre-eclampsia (aOR $3.3,95 \%$ CI 1.2 to 9.4) compared with women without epilepsy (table 3 ).

There was no increased risk of hypertensive complications for any other AEDs used in monotherapy.

The association between early onset pre-eclampsia and WWE disappeared when applying sensitivity analyses for smoking (182 478 women). Sensitivity analyses for BMI $>30 \mathrm{~kg} / \mathrm{m}^{2}$ included 60740 women and the association for any hypertensive disorder disappeared for WWE, while the risk of mild pre-eclampsia increased (aOR 1.8, 95\% CI 1.1 to 2.9). All increased risks for WWE with valproate disappeared when sensitivity analyses 
Table 1 Maternal characteristics

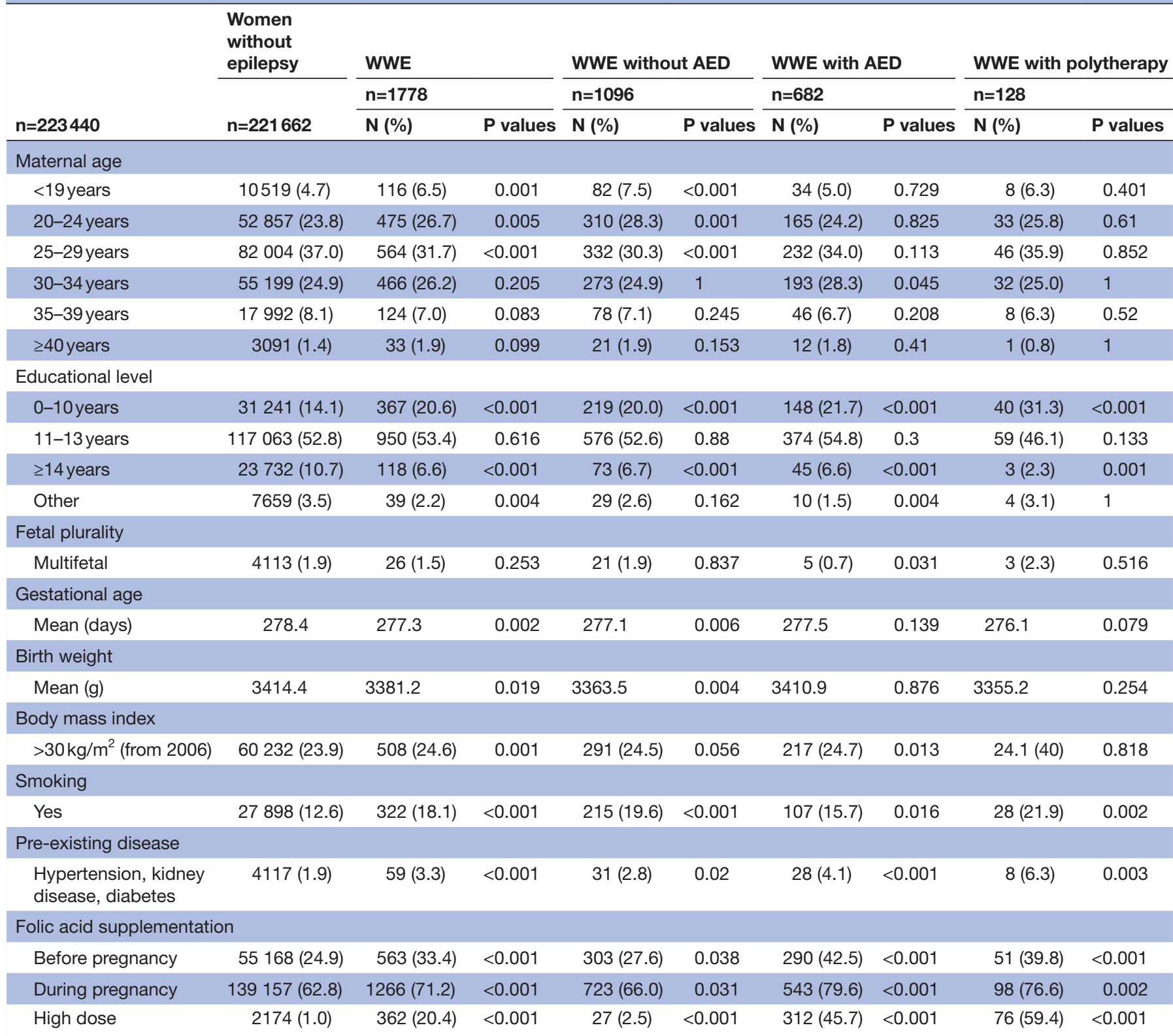

First pregnancies as recorded in Medical Birth Registry of Norway (2004-2012).

AED, antiepileptic drug; WWE, women with epilepsy.

with BMI $>30 \mathrm{~kg} / \mathrm{m}^{2}$ was performed. Separate analyses of women with BMI $>30 \mathrm{~kg} / \mathrm{m}^{2}$ only, showed no significant differences for any of the outcome variables when WWE, WWE with AED and WWE without AED were compared with women without epilepsy. For women with BMI $>30 \mathrm{~kg} / \mathrm{m}^{2}$, WWE with valproate had a higher prevalence of mild pre-eclampsia $(16.7 \%)$ than women without epilepsy $(6.7 \%)$. This difference was not significant, but the number of cases was low. BMI $>30 \mathrm{~kg} / \mathrm{m}^{2}$ and other relevant chronic diseases had no significant interactions with epilepsy in logistic regression analyses. However, these variables had more impact on all hypertensive outcomes than a diagnosis of epilepsy. The impact of standard folic acid and of high-dose folic acid supplementation was examined in all population subcategories.
There were no significant differences among WWE for any of the hypertensive outcomes between use and no use of such supplementations.

\section{DISCUSSION}

We found an increased risk of mild pre-eclampsia in WWE. The specified newer AEDs, lamotrigine and levetiracetam, used in monotherapy did not predispose for this complication. Use of valproate monotherapy was significantly associated with mild pre-eclampsia, in contrast to other AEDs. WWE, with and without AEDs, did not have an increased risk of gestational hypertension, severe pre-eclampsia, early onset pre-eclampsia, HELLP or eclampsia when compared with women without epilepsy. 


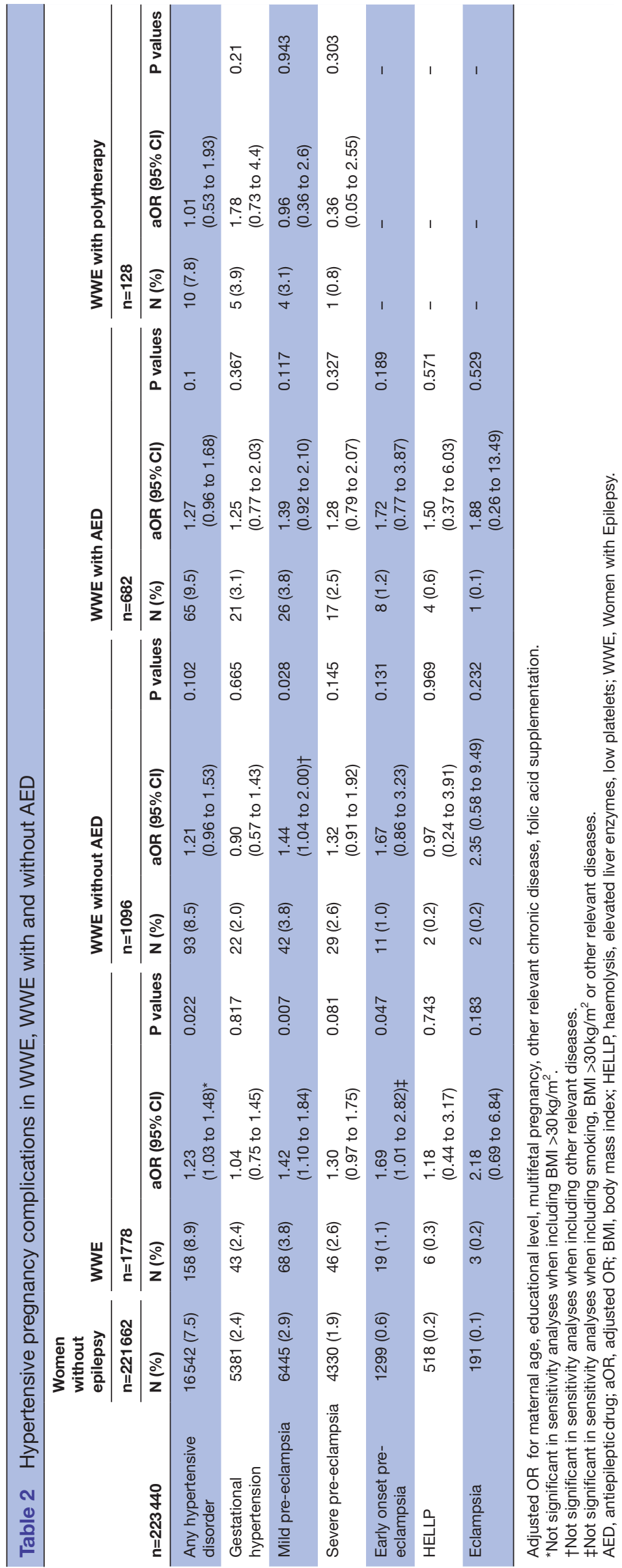






The present study shows that most WWE, and in particular when treated with the newer AEDs, do not develop hypertensive complications. WWE with AED had comparable prevalence of mild pre-eclampsia to WWE without AED, but did not reach significance. This probably reflects marginal differences influenced by sample size. Cardiovascular risk factors (smoking, BMI $>30 \mathrm{~kg} / \mathrm{m}^{2}$, pre-existing kidney disease, hypertension and diabetes) were relevant for hypertensive complications in WWE. The mechanism for the increased risk of hypertensive complications in WWE in first pregnancies could therefore be partly mediated by modifiable external risk factors and comorbidity. The specific risk in WWE on valproate was linked to high BMI. Weight gain and metabolic syndrome represent side effects of valproate. ${ }^{16}$ Our study does not support any additional mechanism for valproate-associated mild pre-eclampsia. When pregnancies with BMI $>30 \mathrm{~kg} / \mathrm{m}^{2}$ were analysed separately, WWE with valproate had a high prevalence of mild pre-eclampsia, but the increased risk was not significant, possibly due to few cases. A previous study showed increased risk of pre-eclampsia in women on valproate, but this was not confirmed by later studies. ${ }^{312}{ }^{13}$ Valproate can induce endocrine changes and polycystic ovary syndrome in WWE and polycystic ovary syndrome is associated with hypertensive pregnancy complications. ${ }^{16}{ }^{20-22}$ Polycystic ovary syndrome is also associated with overweight, so endocrine factors could be important for our findings. Valproate increases the risk of malformations in children exposed during pregnancy. ${ }^{623}$ Because of this, as well as delayed neurodevelopment, The European Medicines Agency has strengthened the advice not to prescribe valproate to WWE in fertile age. ${ }^{24}$ Valproate is therefore not first-line treatment in pregnancy. The observed valproate-associated mild pre-eclampsia could represent bias through confounding by indication, although we would expect valproate to be avoided in women without other risk factors than epilepsy.

The newer AEDs, lamotrigine and levetiracetam, were not associated with increased risk of mild pre-eclampsia, and lamotrigine did not increase hypertensive pregnancy complications in general. A small study has previously reported an increased risk of pre-eclampsia in WWE on lamotrigine. ${ }^{3}$ Lamotrigine has been considered a preferred option for WWE in need of AED treatment because children exposed to lamotrigine in pregnancy have a lower rate of malformations and better cognitive function than after exposure to other AEDs. ${ }^{568}$ The combination of a low rate of adverse fetal effects and no increased risk of hypertensive complications supports the use of lamotrigine in WWE before and during pregnancy. Levetiracetam is a potent, broad-spectrum AED with low rate of adverse fetal outcomes. ${ }^{5}$ Levetiracetam use among pregnant WWE is increasing, but levetiracetam is less studied than lamotrigine and maternal safety during pregnancy is not well documented. ${ }^{5625}$ To our knowledge, there are no 
previous studies on hypertensive complications in WWE with levetiracetam in pregnancy.

Mild, but not more severe pre-eclampsia was increased among WWE. Proactive management and close surveillance of WWE could explain why the mild disease did not progress further. Similarly, the close surveillance of WWE could have increased the detection of mild pre-eclampsia. Ascertainment bias may have influenced other outcomes as well. Alternatively, mild and severe hypertensive complications in pregnancies could have different underlying pathophysiological mechanisms in WWE.

Strengths of our study are that we have analysed first pregnancies only, have specified the types of hypertensive complications and have analysed WWE on specific AEDs in monotherapy only. Parity is a complex variable in reproductive epidemiology and may lead to bias as the risk of hypertensive complications changes significantly in subsequent pregnancies. ${ }^{26-28}$ Our large and unselected nationwide cohort enabled assessment of effects of individual AEDs in monotherapy. Data were linked to the NorPD that showed the exact drug dispensation during pregnancy. The registration and standardised data collection in the national databases that were used to provide information of high validity and include important possible confounders for hypertensive disorders. ${ }^{329}$ The prevalence of WWE in our population was $0.79 \%$ and the incidence of pre-eclampsia was $5.4 \%$, both in accordance with previous population-based studies. ${ }^{124143031}$

A limitation of this study was the possible inclusion of previous and non-active epilepsy, and also of women with undefined seizures in the epilepsy group. This could have increased the total epilepsy population and thereby masking some effects in our group with untreated epilepsy. The MBRN did not provide data on type of epilepsy or seizure activity. However, a small study did not find any association between seizure activity and hypertensive complications in pregnancy. ${ }^{3}$ Women without epilepsy but using AEDs for other conditions were not excluded in our study. This group constituted $0.1 \%$ in the control population and did not affect the outcomes. Psychiatric and pain conditions are the main reasons for AED use in women without epilepsy. NorPD records dispensed medications which may leave room for variation in actual compliance. As $95 \%$ of WWE with AED collected more than one dispensation during their pregnancy, we presume that the patients used their AED as prescribed.

BMI above or below $30 \mathrm{~kg} / \mathrm{m}^{2}$ influenced the results for WWE. MBRN has only registered BMI since 2006, thus, it was not possible to control for this variable in the complete population. A previous study has indicated an increased risk for pre-eclampsia in WWE with BMI $>25 \mathrm{~kg} / \mathrm{m}^{2} .{ }^{32}$ High BMI represents a risk factor for hypertensive disorders and there is a global rise in BMI in young women. ${ }^{33}$ However, a previous study on MBRN data has shown a slight decrease of pre-eclampsia in the time period for our study. ${ }^{30}$ A decrease in pre-eclampsia is most likely also affecting WWE and thereby possibly narrowing the differences between WWE and women without epilepsy.

HELLP, eclampsia and early onset pre-eclampsia are rare hypertensive manifestations. Lack of events in some of our subgroups can be insufficient to prove significance, despite our large sample size. Differences in statistical outcome across treatment subcategories for WWE can similarly have been influenced by sample size. Our study lacks information on ethnicity and marital status, not released by MBRN due to ethical considerations.

\section{CONCLUSION}

Mild pre-eclampsia was the only hypertensive complication to be increased in first pregnancies of WWE. This is of clinical importance for WWE since mild pre-eclampsia, in contrast to severe forms of pre-eclampsia, is not associated with increased maternal or fetal morbidity or mortality in pregnancy. ${ }^{19}$ WWE have previously been considered as high-risk parturients by having a significant increase in several adverse pregnancy outcomes, including hypertensive complications. ${ }^{231011}$ Our study shows that most WWE do not develop hypertensive complications. WWE on the newer AEDs, lamotrigine and levetiracetam in particular, do not have an additional risk for severe hypertensive complications. A healthcare system focusing on risk assessment, prevention of modifiable risk factors and modern treatment options should be the key to a further decrease in hypertensive complications in pregnant WWE.

Acknowledgements This research was kindly supported by the Fund of Torbjørg Hauge's Legacy. We are grateful for data provision and linkage that were performed by Medical Birth Registry of Norway, Norwegian Prescription Database and Statistics Norway.

Contributors KCD conceived and designed the study, analysed the data and drafted the manuscript. IB provided input on design, interpretation and presentation of the study. N-HM provided input on design and analyses, interpretation of analyses and critically revised the manuscript. NEG conceived and designed the study, and critically revised the presentation and discussion of the results in the manuscript. The final manuscript was approved by all authors.

Funding This research was kindly supported by the Fund of Torbjørg Hauge's Legacy.

Competing interests None declared.

Patient consent Not required.

Ethics approval This study was approved by the Regional Ethics Committee (REK 2013/186) and the Norwegian Data Protection Authority.

Provenance and peer review Not commissioned; externally peer reviewed.

Data sharing statement Data from Medical Birth Registry of Norway and Norwegian Prescription Database are available at Norwegian Institute of Public Health.

Open Access This is an Open Access article distributed in accordance with the Creative Commons Attribution Non Commercial (CC BY-NC 4.0) license, which permits others to distribute, remix, adapt, build upon this work non-commercially, and license their derivative works on different terms, provided the original work is properly cited and the use is non-commercial. See: http://creativecommons.org/ licenses/by-nc/4.0/

(c) Article author(s) (or their employer(s) unless otherwise stated in the text of the article) 2018. All rights reserved. No commercial use is permitted unless otherwise expressly granted. 


\section{REFERENCES}

1. Artama M, Gissler M, Malm H, et al. Effects of maternal epilepsy and antiepileptic drug use during pregnancy on perinatal health in offspring: nationwide, retrospective cohort study in Finland. Drug Saf 2013;36:359-69.

2. Borthen I, Eide MG, Veiby G, et al. Complications during pregnancy in women with epilepsy: population-based cohort study. BJOG 2009;116:1736-42.

3. Borthen I, Eide MG, Daltveit AK, et al. Obstetric outcome in women with epilepsy: a hospital-based, retrospective study. BJOG 2011;118:956-65.

4. Richmond JR, Krishnamoorthy P, Andermann E, et al. Epilepsy and pregnancy: an obstetric perspective. Am J Obstet Gynecol 2004;190:371-9.

5. Weston J, Bromley R, Jackson CF, et al. Monotherapy treatment of epilepsy in pregnancy: congenital malformation outcomes in the child. Cochrane Database Syst Rev 2016;11:CD010224.

6. Bromley R, Weston J, Adab N, et al. Treatment for epilepsy in pregnancy: neurodevelopmental outcomes in the child. Cochrane Database Syst Rev 2014;10:CD010236.

7. Gerard EE, Meador KJ. Managing epilepsy in women. Continuum 2016;22:204-26.

8. Tomson T, Xue H, Battino D. Major congenital malformations in children of women with epilepsy. Seizure 2015;28:46-50.

9. Say L, Chou D, Gemmill A, et al. Global causes of maternal death: a WHO systematic analysis. Lancet Glob Health 2014;2:e323-33.

10. MacDonald SC, Bateman BT, McElrath TF, et al. Mortality and morbidity during delivery hospitalization among pregnant women with epilepsy in the United States. JAMA Neurol 2015;72:981-8.

11. Viale L, Allotey J, Cheong-See F, et al. Epilepsy in pregnancy and reproductive outcomes: a systematic review and meta-analysis. Lancet 2015;386.

12. Veiby G, Daltveit AK, Engelsen BA, et al. Pregnancy, delivery, and outcome for the child in maternal epilepsy. Epilepsia 2009;50:2130-9.

13. Pilo C, Wide K, Winbladh B. Pregnancy, delivery, and neonatal complications after treatment with antiepileptic drugs. Acta Obstet Gynecol Scand 2006;85:643-6.

14. Viinikainen K, Heinonen S, Eriksson K, et al. Communitybased, prospective, controlled study of obstetric and neonatal outcome of 179 pregnancies in women with epilepsy. Epilepsia 2006;47:186-92.

15. Razaz N, Tomson T, Wikström AK, et al. Association between pregnancy and perinatal outcomes among women with epilepsy. JAMA Neurol 2017;74:983-91.

16. Belcastro V, D'Egidio C, Striano P, et al. Metabolic and endocrine effects of valproic acid chronic treatment. Epilepsy Res 2013;107:1-8.

17. Vajda FJ, O'Brien T, Lander C, et al. The efficacy of the newer antiepileptic drugs in controlling seizures in pregnancy. Epilepsia 2014;55:1229-34.
18. Nicholas JM, Ridsdale L, Richardson MP, et al. Trends in antiepileptic drug utilisation in UK primary care 1993-2008: cohort study using the General Practice Research Database. Seizure 2012;21:466-70.

19. American College of Obstetricians and GynecologistsTask Force on Hypertension in Pregnancy. Hypertension in pregnancy. Report of the American College of Obstetricians and Gynecologists' Task Force on Hypertension in Pregnancy. Obstet Gynecol 2013;122:1122-31.

20. Hu X, Wang J, Dong W, et al. A meta-analysis of polycystic ovary syndrome in women taking valproate for epilepsy. Epilepsy Res 2011:97:73-82

21. Qin JZ, Pang LH, Li MJ, et al. Obstetric complications in women with polycystic ovary syndrome: a systematic review and meta-analysis. Reprod Biol Endocrinol 2013;11:56.

22. Isojärvi JI, Laatikainen TJ, Pakarinen AJ, et al. Polycystic ovaries and hyperandrogenism in women taking valproate for epilepsy. $N$ Engl J Med 1993;329:1383-8.

23. Tomson T, Battino D, Bonizzoni E, et al. EURAP study group. Dosedependent risk of malformations with antiepileptic drugs: an analysis of data from the EURAP epilepsy and pregnancy registry. Lancet Neurol 2011;10:609-17.

24. Agency EM. CMDh agrees to strengthen warnings on the use of valproate medicines in women and girls: European Medicines Agency 2014. 2017 http://www.ema.europa.eu/ema/index.jsp?curl= pages/news_and_events/news/2014/11/news_detail_002220.jsp\& mid=WC0b01ac058004d5c1.

25. Eurap Study Group. Utilization of antiepileptic drugs during pregnancy: comparative patterns in 38 countries based on data from the EURAP registry. Epilepsia 2009;50:2305-9.

26. Wilcox AJ. Fertility and pregnancy: an epidemiologic perspective. Oxford: Oxford University Press, USA, 2010.

27. Ebbing $\mathrm{C}$, Rasmussen S, Skjaerven R, et al. Risk factors for recurrence of hypertensive disorders of pregnancy, a populationbased cohort study. Acta Obstet Gynecol Scand 2017;96:243-50

28. Hernández-Díaz S, Toh S, Cnattingius S. Risk of pre-eclampsia in first and subsequent pregnancies: prospective cohort study. BMJ 2009;338:b2255

29. Thomsen LC, Klungsøyr K, Roten LT, et al. Validity of the diagnosis of pre-eclampsia in the Medical Birth Registry of Norway. Acta Obstet Gynecol Scand 2013;92:943-50.

30. Klungsøyr K, Morken NH, Irgens L, et al. Secular trends in the epidemiology of pre-eclampsia throughout 40 years in Norway: prevalence, risk factors and perinatal survival. Paediatr Perinat Epidemiol 2012;26:190-8.

31. Abalos E, Cuesta C, Grosso AL, et al. Global and regional estimates of preeclampsia and eclampsia: a systematic review. Eur J Obstet Gynecol Reprod Biol 2013;170:1-7.

32. Kolstad E, Veiby G, Gilhus NE, et al. Overweight in epilepsy as a risk factor for pregnancy and delivery complications. Epilepsia 2016;57:1849-57.

33. WHO. Obesity health topic: World Health Organization;. 2017 http:// www.who.int/topics/obesity/en/ (cited Jun 2017). 